\title{
Utility of Cognitive Function Assessment in Patients Undergoing Surgery on a Third Level Hospital in Mexico City
}

\author{
Andrea Díaz Koirif ${ }^{1,2}$, Alberto Robles Méndez Hernández ${ }^{1,2, *}$, Gabriel Enrique Mejía \\ Terrazas $^{2}$,Lizeth Villalobos Ramírez ${ }^{3}$, María Natalia Denisse García del Mazo ${ }^{2}$, Adriana Díaz \\ Anzaldúa $^{4}$,Francisco Juárez García ${ }^{4}$, Ruth Alcalá Lozano ${ }^{4}$, María Leonor González Arrieta ${ }^{5}$, \\ Alberto Juan Lopez Bascope ${ }^{5}$ \\ ${ }^{1}$ Facultad Mexicana de Medicina, Universidad La Salle México \\ ${ }^{2}$ Hospital Angeles Metropolitano \\ ${ }^{3}$ Hospital Dalinde \\ ${ }^{4}$ Clinical Research Branch, Instituto Nacional de Psiquiatría Ramón de la Fuente Muñiz \\ ${ }^{5}$ Professor of the Anesthesiology course, Hospital Angeles Metropolitano
}

*Corresponding Author: Alberto Robles Méndez Hernández, Facultad Mexicana de Medicina, Universidad La Salle México, Hospital Angeles Metropolitano

\begin{abstract}
Background: Postoperative cognitive dysfunction can be related to surgical processes and applied anesthetic techniques. The incidence of extensive surgery on older patients has increase as the population pyramid transition occurs, so prevention of postoperative cognitive dysfunction should be prioritized.

Material and Methods: A prospective observational study was conducted, which included 47 Mexican subjects between the ages of 45 and 80 years. The MoCA test was applied before and after surgery in a private hospital in Mexico City between December 2018 and June 2019

Results: The Gender distribution was $46.8 \%$ were men and $53.2 \%$ were women, with an average age of 53.9 \pm 8.5 years (range 45-80 years). Cognitive impairment was identified in $63.8 \%$ of patients, men show statistically significant difference between the pre and post-anesthetic attention test.

Conclusions: A larger sample is needed to demonstrate the benefit of the pre-test to make an adjustment of the anesthetic medications, in order to decrease cognitive dysfunction in post-surgical period, our study suggests that pre-anesthetic testing may be beneficial in adjusting anesthesia and reducing postoperative cognitive dysfunction.
\end{abstract}

Key words: cognitive dysfunction, anesthesia, surgery, anesthetics, general anesthesia

\section{INTRODUCTION}

The brain is the main site of action of anesthetic drugs. For many years, it was assumed that their effects did not go beyond their pharmacological action and that the brain was restored to its previous state once the agent was removed. However, there is increasing evidence that supports other notion. There may be long-term and even permanent anatomical and functional changes after the administration of anesthetic drugs. The brain may be more vulnerable at the first and final stages of life.

The term cognition comes from the Latin "cognoscere", which means "to know", and refers to all the brain processes that allow individuals to think, acquire knowledge, solve problems, plan the future and act consciously; it consists of mental processes such as perception, memory, learning, language and thought required for our daily life and it should not be confused with the concept of intelligence. [1]Postoperative cognitive dysfunction (POCD) is defined as a cognitive impairment that arises after a surgical procedure. Its diagnosis requires pre- and postoperative psychometric tests. Its manifestations are multiple and may be subtle or not, depending on the cognitive domains that are affected.[2] There is growing evidence that impaired preoperative brain status, known as cognitive reserve, predicts patients at high risk for developing cognitive deficit.[3] 
Likewise, the surgical process itself and the applied anesthetic techniques could have an enormous influence on the evolution. Since the 19th century, there have been reports that describe behavioral changes after anesthesia. In 1955 , Bedford affirmed that more than $10 \%$ of those who underwent surgery under general anesthesia had some degree of mental deterioration, and cognitive dysfunction.[4]

The use of neuropsychological tests in the 1980s allowed to establish a quantitative basis for measuring the initial cognitive function of the patients and thus determining the presence of cognitive impairment. This facilitated the quantitative measure of cognitive dysfunction. Krenk et al. noted in their review that POCD can occur at any age, but tends to last longer and affects more severely the daily activities of patients over 60 years old.[5] Monk and colleagues reported the presence of POCD at hospital discharge in $36.6 \%$ of surgical patients between 18 and 39 years of age, $30.4 \%$ of those between 40 and 59 years, and $41.4 \%$ of those over 60 years.[6]In the International Study of Postoperative Cognitive Dysfunction (ISPOCD), it was shown that on the seventh day of the postoperative period, $25.8 \%$ of 1,011 patients presented POCDvs $3.4 \%$ of 176 patients in the control group. At 3 months of follow-up, of 910 patients, $9.9 \%$ presented POCD, as opposed to $2.8 \%$ in the control group.[7]

POCD occurs more frequently during cardiac and orthopedic surgeries, as a response to surgical stress, which may be one of the possible mechanisms leading to POCD. The surgical procedure could cause a systemic response to stress, the release of neuroendocrine hormones, and an inflammatory cytokine response; as a result, there may be changes in brain function in associated with POCD. Another probable mechanism is neuro- degeneration due to the apoptotic process.[8]

There is a hypothesis stating that vascular risk factors are a modifiable key to the development of dementia and cognitive disorders. Together with the usual pre-anesthetic assessments, the usefulness of the Cardiovascular Risk Factors, Aging and Dementia (CAIDE) score has been demonstrated, which predicts cognitive impairment with greater certainty, especially dementia.

The CAIDE risk score was developed to predict dementia in the elderly, based on risk factors during adulthood. Its components are age, education, sex, systolic blood pressure, body mass index, total cholesterol, physical activity and the APOE genotype $\Sigma$ 4.[9]

An increase in age, a prolonged duration of anesthesia, a low level of education, the length of the surgery, a second operation, depression, mild cognitive impairment, the presence of a heart attack, postoperative infections, and respiratory complications have been considered as risk factors for the development ofearly POCD.[10]

In the most extensive identified study comparingregional anesthesia (RA) and general anesthesia (GA), the incidence of POCDone week after the surgery was $19.7 \%$ after GA and $12.5 \%$ after RA; however, this difference was not a statistically significant. It has also been suggested that the type of anesthesia does not have a long-term impact on cognitive evolution; nevertheless, it is noteworthy that the statistics used havevaried and that the repeated use of sedation and opioids during RA has been constant,[11]

The Montreal Cognitive Assessment (MoCA) is a brief screening test, designed to aid in the detection of mild cognitive impairment (MCI) and dementia. Its psychometric characteristics describe a high level of reliability and validity, with a sensitivity of $87 \%$ and a specificity close to $90 \%$ for MCI, with a cut-off point <18.(26)It consists of a battery of short tests, of easy administration, with an approximate duration of 10 minutes. The purpose of MoCA is to detect the presence of DCL and differentiate it from cognitive alterations consequent to normal aging. It evaluates executive function, visuospatial ability, memory, attention, concentration and working memory, language, and orientation; the maximum score is 30 points.[12, 13]

\section{METHODS}

A prospective observational study was conducted, which included 47 Mexican subjects between the ages of 45 and 80 years. The aims and procedures were explained to each participant, and all of them signed an informed consent; the MoCA test was applied before and after surgery in a private hospital in Mexico City between December 2018 and June 2019. An initial evaluation was carried out including socio-demographic and disease clinical data collection through a format designed for this purpose, with a direct interview with the patient and his/her relatives. 
The study was approved by the Ethics and Research Committees of the private hospital. Participation of the subjects was voluntary, and the identification of participants and relatives was kept confidential. Chi square and $t$ test were also used.

The database was performed, and the statistical program SPSS version 23.0 for Mac IOS 10.6 was used to perform statistical data analysis. Clinical and demographic characteristics were described as frequencies and percentages for the categorical variables and as means and standard deviations (S.D.) for the continuous variables using $\mathrm{X} 2$ test.

\section{Results}

A total of 47 patients agreed to participate and were included in the study. They all underwent surgery at a private hospital in Mexico City. From them, $46.8 \%$ were men and $53.2 \%$ were women, with an average age of $53.9 \pm 8.5$ years (range 45-80 years) and an education of $13 \pm 4.1$ years (range six to 18 years), equivalent to the first year of the bachelor's degree. Among the Table1: Results of MoCA instrument for cognitive function, pre-anesthesia and post-anesthesia

\begin{tabular}{|l|l|l|l|l|l|l|}
\hline & $\begin{array}{c}\text { Female } \\
\text { Mean }\end{array}$ & $\begin{array}{c}\text { Female } \\
\text { SD }\end{array}$ & $\begin{array}{c}\text { Male } \\
\text { Mean }\end{array}$ & Male SD & $\begin{array}{c}\text { Total } \\
\text { Mean }\end{array}$ & Total SD \\
\hline Pre-anvisuospatial & 4 & 1.2 & 4 & 1.3 & 4 & 1.2 \\
\hline Post-an visuospatial & 3.6 & 1.6 & 4 & 1.3 & 3.8 & 1.4 \\
\hline Pre-annaming & 3 & 0.2 & 2.9 & 0.3 & 2.9 & 0.2 \\
\hline Post-annaming & 3 & 0.2 & 2.9 & 0.3 & 2.9 & 0.2 \\
\hline Pre-an attention & 4.6 & 1.4 & 4.5 & 1.1 & 4.6 & 1.2 \\
\hline Post-an attention & 4.4 & 1.3 & 4.1 & 1.4 & 4.3 & 1.3 \\
\hline Pre-anlanguage & 2.3 & 0.9 & 2.4 & 0.9 & 2.3 & 0.9 \\
\hline Post- an language & 2.2 & 0.9 & 2.3 & 0.8 & 2.2 & 0.8 \\
\hline Pre-anabstraction & 1.6 & 0.5 & 1.9 & 0.4 & 1.7 & 0.4 \\
\hline Post-anabstraction & 1.7 & 0.5 & 1.9 & 0.3 & 1.8 & 0.4 \\
\hline Pre-andelayedrecall & 2.5 & 1.9 & 1.7 & 1.6 & 2.1 & 1.8 \\
\hline Post-andelayedrecall & 2.6 & 2 & 1.9 & 1.6 & 2.3 & 1.8 \\
\hline Pre-anorientation test & 5.6 & 0.8 & 5.5 & 1 & 5.5 & 0.9 \\
\hline Post-anorientation test & 5.4 & 0.9 & 5.4 & 1 & 5.4 & 0.9 \\
\hline Pre-an Total MOCA score & 23.6 & 3.5 & 22.8 & 3 & 23.2 & 3.3 \\
\hline Post-an total MOCA score & 22.9 & 4.1 & 22.5 & 4 & 22.7 & 4 \\
\hline PRE-An: Pre-anesthesia test, Post-An test: Post-anesthesia & & & & \\
\hline
\end{tabular}

\section{DISCUSSION}

The search for factors that help evaluate changes in cognitive functions remains a premise in clinical research. Several reports have described a close relationship between cognitive function and concomitant diseases.In our study, six out of ten patients had cognitive impairment, regardless of a diagnosis of hypertension or diabetes.

In the tests, the most frequently identified errors were on the cube and the clock drawing, concomitant pathologies, $23.4 \%$ were diabetic and $36.2 \%$ were hypertensive.

By type of surgery, $72.3 \%$ were treated by general surgery and $27.7 \%$ by orthopedics. According to the type of administered anesthesia, 76.6\% was general anesthesia and $23.4 \%$ regional anesthesia. There were no statistically significant differences between de group of general anesthesia and regional anesthesia regarding sex $(\mathrm{p}=0.20)$, age $(\mathrm{p}=0.24)$, years of education $(\mathrm{p}=0.67)$, presence of diabetes $(\mathrm{p}=0.42)$, or presence of hypertension $(\mathrm{p}=0.63)$.

We were not able to determine an association between the use of general or regional anesthesia and changes in cognitive function. Nevertheless, according to the cut-off point suggested by the authors of the MoCA instrument (cut-off point of 26) and with an adjustment made by the subjects' education (increase of one point in the total grade when education is $<12$ years), $63.8 \%$ of patients presented cognitive impairment table 1. subtraction, serial attention, verbal fluency and abstraction, and they were especially common in patients with less years of education, as established in other studies [14]. In addition, these patients presented a clearer decrease in the postoperative test than those who had more years of education. Thus, patients with less schooling may be more labile to the anesthetic effects on the brain or those who have more schooling may be more likely to compensate for deficiencies. We believe that the one or twopoint decrease in all the preoperative tests may 
be due to the anxiety that causes undergoing a surgical procedure. However, in the postoperative test there was a decrease of more than four points that proves that there may bea mild cognitive dysfunction in some patients, even when this was not detected by the test.

\section{CONClusions}

It is needed a larger sample to demonstrate the benefit of the pre-test to make an adjustment of the anesthetic medications. Preliminarilypretesting cognitive function to make an adjustment of anesthetic medications is helpful to decrease cognitive dysfunction in post-surgical period.

\section{REFERENCES}

[1] Hanning, C.D., Postoperative cognitive dysfunction. Br J Anaesth, 2005. 95(1): p. 82-7.

[2] Rundshagen, I., Postoperative cognitive dysfunction. Dtsch Arztebl Int, 2014. 111(8): p. 119-25.

[3] Monk, T.G. and C.C. Price, Postoperative cognitive disorders. Curr Opin Crit Care, 2011. 17(4): p. 376-81.

[4] Bedford, P.D., Adverse cerebral effects of anaesthesia on old people. Lancet, 1955.269 (6 884): p. 259-63.

[5] Krenk, L., L.S. Rasmussen, and H. Kehlet, New insights into the pathophysiology of postoperative cognitive dysfunction. Acta Anaesthesiol Scand, 2010. 54(8): p. 951-6.

[6] Monk, T.G., B.C. Weldon, C.W. Garvan, et al, Predictors of cognitive dysfunction after major noncardiac surgery. Anesthesiology, 2008. 108(1): p. 18-30.

[7] Moller, J.T., P. Cluitmans, L.S. Rasmussen, et al, Long-term postoperative cognitive dysfunction in the elderly ISPOCD1 study. ISPOCD investigators. International Study of
Post-Operative Cognitive Dysfunction. Lancet, 1998. 351(9106): p. 857-61.

[8] Bilotta, F., A. Doronzio, E. Stazi, et al, Postoperative cognitive dysfunction: toward the Alzheimer's disease pathomechanism hypothesis. J Alzheimers Dis, 2010. 22 Suppl 3: p. 81-9.

[9] Kaffashian, S., A. Dugravot, A. Elbaz, et al, Predicting cognitive decline: a dementia risk score vs. the Framingham vascular risk scores. Neurology, 2013. 80(14): p. 1300-6.

[10] Xiao, Q.X., Q. Liu, R. Deng, et añ, Postoperative cognitive dysfunction in elderly patients undergoing hip arthroplasty. Psychogeriatrics, 2020.

[11] Bilotta, F., E. Qeva, and I. Matot, Anesthesia and cognitive disorders: a systematic review of the clinical evidence. Expert Rev Neurother, 2016. 16(11): p. 1311-1320.

[12] Freitas, S., M.R. Simoes, L. Alves, et al, Montreal cognitive assessment: validation study for mild cognitive impairment and Alzheimer disease. Alzheimer Dis Assoc Disord, 2013. 27(1): p. 37-43.

[13] Gil, L., C. Ruiz de Sanchez, F. Gil, et al, Validation of the Montreal Cognitive Assessment (MoCA) in Spanish as a screening tool for mild cognitive impairment and mild dementia in patients over 65 years old in Bogota, Colombia. Int J Geriatr Psychiatry, 2015. 30(6): p. 655-62.

[14] Aguilar-Navarro, S.G., A.J. MimenzaAlvarado, A.A. Palacios-Garcia, et al, Validity and Reliability of the Spanish Version of the Montreal Cognitive Assessment (MoCA) for the Detection of Cognitive Impairment in Mexico. Rev Colomb Psiquiatr, 2018. 47(4): p. 237-243.

Citation: Andrea Díaz Koirif, Alberto Robles Méndez Hernández. Utility of Cognitive Function Assessment in Patients Undergoing Surgery on a Third Level Hospital in Mexico City. ARC Journal of Anesthesiology. 2020; 5(1): 15-18. DOI: dx.doi.org/10.20431/2455-9792.0501004.

Copyright: (C) 2020 Authors. This is an open-access article distributed under the terms of the Creative Commons Attribution License, which permits unrestricted use, distribution, and reproduction in any medium, provided the original author and source are credited. 